\title{
Red Blood Cell Distribution Width in Combination with Serological Markers can Help in the Differentiation between Crohn's Disease and Ulcerative Colitis
}

\author{
Klaudia Farkas ${ }^{1}$, Mária Papp ${ }^{2}$, Tibor Nyári ${ }^{3}$, Ferenc Nagy ${ }^{1}$, Zoltán Szepes ${ }^{1}$, Tibor Wittmann ${ }^{1}$ \\ and Tamás Molnár ${ }^{1, *}$ \\ ${ }^{I}$ Department of Medicine, University of Szeged, Szeged, Hungary \\ ${ }^{2}$ Department of Medicine, University of Debrecen, Debrecen, Hungary \\ ${ }^{3}$ Department of Medical Informatics, University of Szeged, Szeged, Hungary
}

\begin{abstract}
Background: Recent data suggest that red blood cell distribution width (RDW) could be a reliable marker in differentiating Crohn's disease (CD) from ulcerative colitis (UC).

Aim: Our study was to determine the sensitivity and specificity of RDW and serological markers for inflammatory bowel diseases (IBD), the risk for the diseases in case of abnormal RDW value and positive serological markers separately and conjointly and to evaluate the efficacy of RDW in the differential diagnosis between IBD and irritable bowel syndrome (IBS). We also examined the influence of the drug treatments on the alteration of RDW values.

Patients and methods: The clinical records of $98 \mathrm{CD}, 81 \mathrm{UC}, 26$ IBS patients and 84 healthy controls were reviewed. Sensitivity, specificity and relative risk of RDW and ASCA IgA, ASCA IgG and anti-OmpC antibodies for CD and UC were assessed. RDW values of IBS patients and IBD patients were also statistically compared.

Results: RDW was significantly elevated in IBD patients compared to IBS patients. 92\% specificity cutpoint at a RDW level of $13.4 \%$ was detected in both $\mathrm{CD}$ and UC independently of the disease activity. Significantly higher risk for CD was observed in case of combined assessment of abnormal RDW and positive ASCA IgA vs. the other serological markers or to separate determination of RDW.

Conclusion: RDW could be a useful and inexpensive activity marker, which may help in the differential diagnosis between IBD and IBS and using in combination with ASCA IgA it shows a synergistic effect in the distinction of CD from UC.
\end{abstract}

Keywords: Red blood cell distribution width, Crohn's disease, ulcerative colitis.

\section{INTRODUCTION}

The term indeterminate colitis is used for that $10-15 \%$ of inflammatory bowel disease (IBD - Crohn's disease [CD], ulcerative colitis [UC]) cases in which exact diagnosis of $\mathrm{CD}$ or UC cannot be established, neither with endoscopy, nor with histology [1]. The differentiation between CD and UC is important to plan the therapeutical strategy especially in case of severe colitis requiring surgical intervention. In the lack of an accurate differential diagnostic marker, in most of the indeterminate colitis cases definitive diagnosis is usually made on later follow up. Despite serological markers being more and more available, their low sensitivity and relatively high cost limit their role in the distinction between the two forms of IBD. For the differentiation between $\mathrm{CD}$ and $\mathrm{UC}$, the combined determination of ASCA IgG and pANCA proved to be the most accurate [2]. The importance of the

*Address correspondence to this author at the Department of Medicine, University of Szeged, H-6720, Korányi Alley 8, Szeged, Hungary;

Tel: +36-62-545186; Fax: +36-62-545185;

E-mail: mot@in1st.szote.u-szeged.hu distinction between $\mathrm{CD}$ and $\mathrm{UC}$ motivates the clinicians to search for new, possibly cost-effective and simple differential diagnostic markers.

Clarke et al. have published a study about the potential role of red blood cell distribution width (RDW) in differentiating CD from UC for a year [3]. RDW, which represents the size variability within the red blood cell population, is reported as part of a standard complete blood count, which is a routine method in monitoring IBD patients. It helps to classify the different types of anemia and may be increased even before iron deficiency becomes obvious. Elevated RDW values with normal hemoglobin levels are reported to be the most frequent haematochemical abnormalities in celiac disease $[4,5]$, although there has been limited data dealing with its usefulness in IBD.

In the view of the hypothesis of Clarke et al., we have also examined, whether RDW could help to differentiate CD from UC both in active state and in remission, and whether there is a correlation between RDW and the activity of IBD [6]. On the basis of our results RDW may differentiate be- 
tween the inactive form of $\mathrm{UC}$ and $\mathrm{CD}$, but not in case of activity, when it would be more important. Cakal et al. [7] have recently found RDW to be the most sensitive and specific parameter among other inflammatory laboratory parameters (C-reactive protein, fibrinogen level, erythrocyte sedimentation rate, leukocyte and platelet counts) for detecting active UC but not active CD. However, it should be noted that only 22 patients were enrolled in the $\mathrm{CD}$ group.

Considering the earlier findings and our results, questions have raised whether RDW could differentiate not only between IBD and healthy controls, but between IBD and irritable bowel syndrome (IBS) as well and whether drug therapy could have an influence on the alteration of RDW values. Furthermore, it is also interesting and questionable, whether the relatively low sensitivity of serological markers could increase their diagnostic sensitivity using them in combination with RDW.

The aim of this study was to assess the sensitivity of RDW in combination with the serological markers in differentiating IBD and to determine their risk for IBD. We also examined, whether the alterations of the RDW values are influenced by the different drug treatments of IBD and whether it could help in the differentiation between IBD and IBS.

\section{PATIENTS AND METHODS}

179 IBD [98 CD (62 females, 36 males), 81 UC (43 females, 38 males)] and 84 age and gender-matched healthy control patients (50 females, 34 males) were enrolled in the study. The control subjects did not have any gastrointestinal disease and were selected from consecutive blood donors in Debrecen. Serological markers such as ASCA IgA, ASCA IgG and anti-OmpC antibodies and RDW values were measured in all patients. Sensitivity and specificity of serological markers and RDW values were assessed by receiver operating characteristic (ROC) analysis. Odds ratios (OR) for IBD in case of abnormal RDW values and positive serological markers were calculated by logistic regression both individually and conjointly.

RDW values of 26 IBS patients [12 patients with IBS-C, 14 with IBS-D (21 females, 5 males)] and the IBD patients were compared by one-way ANOVA with Bonferroni post hoc test. Clinical data of the patients enrolled in the study are presented in Table $\mathbf{1 .}$
Ethical approvals for the study have been obtained from the Ethical Committee of the University of Szeged.

\section{RESULTS}

Sensitivity and specificity of ASCA IgA, ASCA IgG and anti-OmpC antibodies were assessed in CD and UC patients. Specificity of more than $80 \%$ and overall sensitivity of ASCA IgA of $64.3 \%$, ASCA IgG of $51 \%$ and anti-OmpC antibody of $43 \%$ were detected in patients with CD. In UC patients, low sensitivity and high specificity was measured regarding the same serological markers. Sensitivity and specificity of RDW was only $61 \%$ and $64 \%$ in CD patients; $46 \%$ and $64 \%$ in UC patients. Proportion of the sensitivity, specificity and positive predictive value of ASCA IgA, ASCA IgG, anti-OmpC antibody and RDW values in CD and UC are presented in details in Table 2.

Results of logistic regression analysis indicated that patients with negative ASCA IgA/elevated RDW and those with negative ASCA IgG/elevated RDW were at increased risk for CD [OR: 4.09 (95\% CI 1.51-11.08), $\mathrm{p}=0.006$ and OR: 2.94 (95\% CI 1.51-11.08), p=0.004]. Elevated RDW with positive ASCA IgA was strongly associated with CD [OR: 79.4 (95\% CI 9.99-631.64), p<0.0001]. Elevated RDW with positive ASCA IgG showed an OR of 28.28 [(95\% CI 9.99-631.64), $\mathrm{p}<0.0001]$ in CD.

Normal value of RDW is defined between 11-14\%. Elevated RDW values were detected in only $8.3 \%$ of IBS patients. RDW was significantly increased in both active and inactive CD and UC patients comparing to patients with IBS $(\mathrm{p}<0.001$ in active and inactive $\mathrm{CD}, \mathrm{p}<0.0018$ in inactive UC, $\mathrm{p}<0.002$ in active UC).

ROC analysis of RDW values showed 92\% specificity cutpoint at a RDW level of $13.4 \%$ in both CD and UC independently of the disease activity (Fig. 1). The sensitivities were $68 \%$ in inactive $\mathrm{CD}, 78 \%$ in active $\mathrm{CD}, 49 \%$ in inactive $\mathrm{UC}$ and $72 \%$ in active UC at this cutpoint.

5-ASA was used in $46.7 \%$ of CD patients and in $62.7 \%$ of UC patients, azathioprine in $64 \%$ of $\mathrm{CD}$ cases and in $50.7 \%$ of UC cases for maintaining remission. Corticosteroid was added in $33.3 \%$ of CD and in $41.5 \%$ of UC in case of flare ups. RDW levels were significantly increased in patients taking corticosteroids in the inactive $\mathrm{CD}(\mathrm{p}=0.014)$ and in the inactive $\mathrm{UC}$ groups $(\mathrm{p}=0.003)$. In inactive $\mathrm{UC}$, abnor-

Table 1. Clinical Characteristics and the Values of the Serological Markers and RDW in Patients Enrolled in the Study

\begin{tabular}{|c|c|c|c|c|}
\hline Mean age currently (years) & 38.1 & 46.9 & 37 & 55.5 \\
\hline Gender (male/female) & $36 / 62$ & $38 / 43$ & $34 / 50$ & $4 / 21$ \\
\hline ASCA IgA positivity (\%) & 64.3 & 21 & 6.9 & - \\
\hline Anti-OmpC antibody (\%) & 42.9 & 22.2 & 16.3 & - \\
\hline Elevated RDW level (\%) & 63.3 & 45.7 & 35.7 & 8.3 \\
\hline
\end{tabular}


Table 2. Proportion of the Sensitivity, Specificity and Positive Predictive Value of ASCA IgA, ASCA IgG, Anti-OmpC Antibody and RDW Values in CD and UC

\begin{tabular}{|c|c|c|c|}
\hline CD & Sensitivity & Specificity & PPV \\
\hline ASCA IgA & $64.3 \%$ & $93 \%$ & $95.5 \%$ \\
\hline ASCA IgG & $51 \%$ & $94 \%$ & $90.9 \%$ \\
\hline anti-OmpC antibody & $43 \%$ & $83.7 \%$ & $85.7 \%$ \\
\hline RDW & $61 \%$ & $64 \%$ & $67.4 \%$ \\
\hline \multicolumn{4}{|l|}{ UC } \\
\hline ASCA IgA & $21 \%$ & $93 \%$ & $85 \%$ \\
\hline ASCA IgG & $9 \%$ & $94 \%$ & $58.3 \%$ \\
\hline anti-OmpC antibody & $22.2 \%$ & $83.7 \%$ & $72 \%$ \\
\hline RDW & $45.7 \%$ & $64.3 \%$ & $55.2 \%$ \\
\hline
\end{tabular}

PPV: positive predictive value.

mal RDW values were also detected in patients being on azathioprine therapy $(\mathrm{p}=0.007)$.

\section{ROC Curve}

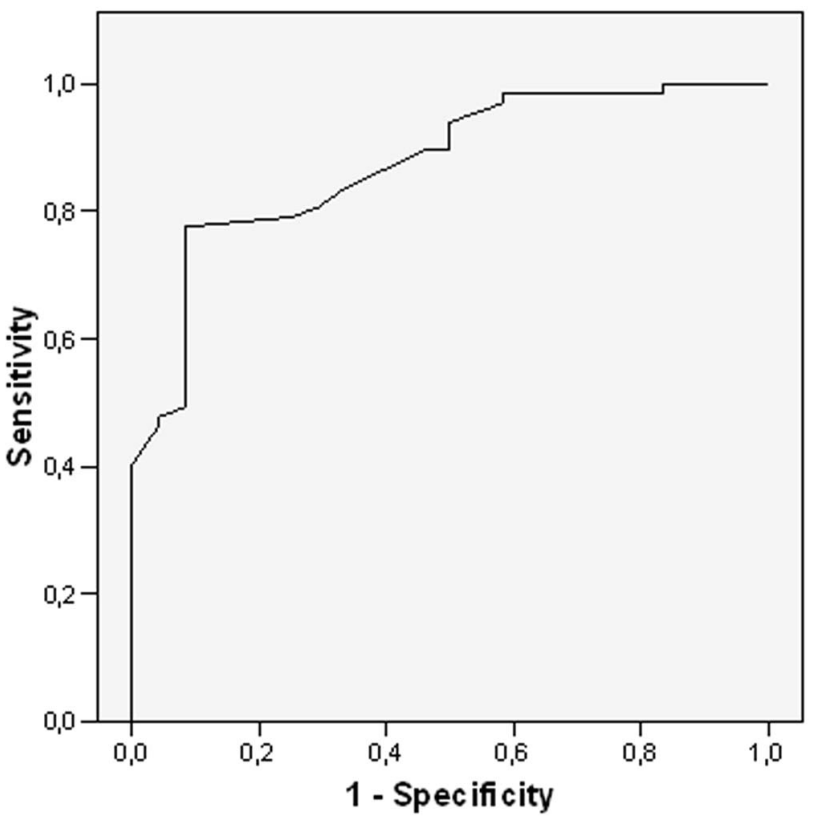

Diagonal segments are produced by ties.

Fig. (1). ROC curve analysis in active CD with a $92 \%$ specificity and $78 \%$ sensitivity cutpoint at RDW of $13.4 \%$

\section{DISCUSSION}

In almost $15 \%$ of IBD cases it is still difficult to differentiate between $\mathrm{CD}$ and UC with the known diagnostic methods. Because surgical procedures are completely and medical therapy is moderately different in the two types of the disease, any noninvasive, widely available test, which provides additional information for the precise differential diagnosis between the two types of IBD, might be very important. The sensitivities of the two main IBD serological markers, pANCA and ASCA are too low to be used as the only diagnostic tools, although using them in combination improves their diagnostic value. A recent study has suggested that RDW could also be important in the differentiation of CD and UC [3]. According to the results of our previous study [6], mean RDW elevated significantly in the active form of both $\mathrm{CD}$ and UC compared to the normal RDW value (CD: $\mathrm{p}=0.0019$, UC: $\mathrm{p}=0.0263$ ). We found significantly increased RDW in inactive CD vs. inactive UC (average RDW values; CD: 14.3 vs. UC: $13.8, p=0.05$ ), while significant correlation could not be detected between the active diseases (average RDW values; $C D: 14.7$ vs. UC: $14.4, \mathrm{P}=0.393$ ). These previous data have revealed that RDW might have a relatively low value in the differentiation of the two forms of IBD, mainly in the inactive period, when it does not have strong influence on therapeutic decisions in contrast with the active phase of the disease.

Although our results did not reveal significant correlation between RDW values in the active period of IBD, elevated mean RDW values in the active form of both $C D$ and UC compared to the normal RDW range, refers that RDW can be a possible activity marker in IBD. Combined use of serological markers and RDW in order to increase their diagnostic sensitivity has not been examined before.

RDW is mainly used in the differential diagnosis of anaemia, particularly in distinguishing iron deficiency from thalassemia and megaloblastic anaemia and other causes of macrocytosis. Elevated RDW reveals anisocytosis even when the mean corpuscular value (MCV) is normal [8]. The overall prevalence of anemia in IBD ranges from $8.8 \%$ to $73.7 \%$ and mainly develops due to iron deficiency and chronic intestinal bleeding, two common complications of IBD [9]. In our previous study increased RDW correlated significantly with the low values of serum iron level in both $\mathrm{CD}$ and UC patients with inactive disease but not in the active form independently of the type of the maintenance treatment [6]. 
Increased RDW levels observed by us in the inactive stage of both type of IBD, suggest that the normal level of the activity indices do not rule out subclinical inflammation and/or iron dysbalance, which completely manifests only in the active phase. RDW of $13.4 \%$ seems to be a value below which IBD can be excluded with $92 \%$ confidence.

We examined the alteration of RDW in patients with IBS and found that RDW levels were within the normal range in the majority of the IBS cases contrary to IBD. Corticosteroid and azathioprine treatment did not influence RDW values in the active form of IBD, the higher RDW levels measured in steroid and immunosuppressive treated IBD patients in remission may reflect on the more severe, complicated disease course or subclinical activity, which does not reflect in the clinical activity indices.

Our results showed markedly high [OR: 79.4 (95\% CI 9.99-631.64), $\mathrm{p}<0.0001]$ risk for $\mathrm{CD}$ in case of elevated RDW value and positive ASCA IgA. Due to the low sensitivity and specificity of RDW, it cannot be the diagnostic magic bullet, but using it in combination with ASCA IgA increases its benefit significantly. Significant prevalence of the abnormal RDW values in active CD and UC can assist clinicians in diagnosing IBD and in differentiating them from IBS or theoretically from other non-inflamed condition. Furthermore, since noninvasive methods are more favorable in the diagnosis of childhood IBD, the assessment of RDW and ASCA IgA together could be practical in these cases.

\section{ABBREVIATIONS}

ANCA $=$ Anti-neutrophil cytoplasmic antibodies

anti-OmpC $=$ Antibody to Escherichia coli outer membrane porin

ASCA $=$ Anti-Saccharomyces cerevisiae antibodies

$\mathrm{CD}=$ Crohn's disease

IBD $=$ Inflammatory bowel diseases

$\begin{array}{ll}\text { IBS } & =\begin{array}{l}\text { Irritable bowel syndrome } \\ \text { IBS-C }\end{array} \\ \text { IBS-D } & =\begin{array}{l}\text { Constipation predominant irritable bowel } \\ \text { syndrome }\end{array} \\ \text { MCV } & =\text { Meandrome corpuscular value } \\ \text { OR } & =\text { Odds ratio } \\ \text { RDW } & =\text { Red blood cell distribution width } \\ \text { ROC } & =\text { Receiver operating characteristic } \\ \text { UC } & =\text { Ulcerative colitis }\end{array}$

\section{ACKNOWLEDGEMENT}

This work was supported by OTKA (CK 80136).

\section{REFERENCES}

[1] Guindi M, Riddell RH. Indeterminate colitis. J Clin Pathol 2004; 57: 1233-44.

[2] Ferrante M, Henckaerts L, Joossens M, et al. New serological markers in inflammatory bowel disease are associated wit complicated disease behaviour. Gut 2007; 56: 1394-403.

[3] Clarke K, Sagunarthy R, Kansal S. RDW as an Additional Marker in Inflammatory Bowel Disease/Undifferentiated Colitis. Dig Dis Sci 2008; 53: 2521-3.

[4] Guidetti CS, Scaglione N, Martini S. Red cell distribution width as a marker of celiac disease: a prospective study. Eur J Gastroenterol Hepatol 2002; 14: 177-81.

[5] Brusco G, Stefani MD, Corazza GR. Increased red cell distribution width and coelic disease. Digest Liver Dis 2000; 32: 128-30.

[6] Molnar T, Farkas K, Szepes Z, et al. RDW can be a useful additional marker in diagnosis Crohn's disease and ulcerative colitis. Dig Dis Sci 2008; 53: 2828-9.

[7] Cakal B, Akoz AG, Ustundag Y, et al. Red cell distribution width for assessment of activity of inflammatory bowel disease. Dig Dis Sci 2009; 54: 842-7.

[8] Evans TC, Jehle D. The red blood cell distribution width. J Emerg Med 1991; 9: 71-4.

[9] Giannini S, Martes C. Anemia in inflammatory bowel disease. Min Gastroenterol Dietol 2006; 52: 275-91. 